\title{
Belief-Assisted Pricing for Dynamic Spectrum Allocation in Wireless Networks with Selfish Users
}

\author{
Zhu Ji and K. J. Ray Liu \\ Electrical and Computer Engineering Department and Institute for Systems Research \\ University of Maryland, College Park, MD 20742 \\ email: zhuji, kjrliu@umd.edu
}

\begin{abstract}
In order to fully utilize the scarce spectrum resources, with the development of cognitive radio technologies, dynamic spectrum allocation becomes a promising approach to increase the efficiency of spectrum usage. In this paper, we consider the spectrum allocation in wireless networks with multiple selfish legacy spectrum holders and unlicensed users as multi-stage dynamic games. A belief-assisted dynamic pricing approach is proposed to optimize overall spectrum efficiency while keeping the participating incentives of the users based on double auction rules. Moreover, considering the budget constraints of the unlicensed users, a dynamic programming approach is further developed to optimize the spectrum allocation over time. The simulation results show that our proposed scheme not only approaches optimal outcomes with low overhead compared to general continuous double auction mechanisms, but also fully exploits the time diversity of spectrum resources when budget constraints exist.
\end{abstract}

\section{INTRODUCTION}

Recently, regulatory bodies like the Federal Communications Commission (FCC) in the United States are recognizing that current static spectrum allocation can be very inefficient considering the bandwidth demands may vary highly along the time dimension or the space dimension. In order to fully utilize the scarce spectrum resources, with the development of cognitive radio technologies, dynamic spectrum access becomes a promising approach to increase the efficiency of spectrum usage, which allows unlicensed wireless users to dynamically access the licensed bands from legacy spectrum holders based on leasing agreements.

Cognitive radio technologies have the potential to provide the wireless devices with various capabilities, such as frequency agility, adaptive modulation, transmit power control and localization. The advances of cognitive radio technologies make more efficient and intensive spectrum access possible on a negotiated or an opportunistic basis. The FCC began to consider more flexible and comprehensive use of available spectrum in [1], [2]. Then, great attentions have been drawn to explore the open spectrum systems [3], [4] for dynamic spectrum sharing. Traditionally, network-wide spectrum assignment is carried out by a central server, namely, spectrum broker [5], [6]. Recently, distributed spectrum allocation approaches [7], [8] have been well studied to enable efficient spectrum sharing only based on local observations. In [7], local bargaining mechanism was introduced to distributively optimize the efficiency of spectrum allocation and maintain bargaining fairness among secondary users. In [8], the authors proposed a repeated game approach to increase the achievable rate region of spectrum sharing, in which the spectrum sharing strategy can be enforced by the Nash Equilibrium of dynamic games. Moreover, efficient spectrum sharing has also been studied from a practical point of view, such as in [9] and [10], which analyzed spectrum sharing games for WiFi networks and cellular networks, respectively.

Although the existing dynamic spectrum access schemes have achieved some success on enhancing the spectrum efficiency and distributive design, most of them focus on efficient spectrum allocation given fixed topologies and cannot quickly adapt to the dynamics of wireless networks due to node mobility, channel variations or varying wireless traffic. Furthermore, existing cognitive spectrum sharing approaches generally assume that the network users will act cooperatively to maximize the overall system performance, which is a reasonable assumption for traditional emergency or military situations. However, with the emerging applications of mobile ad hoc networks envisioned in civilian usage, the users may not serve a common goal or belong to a single authority, which requires that the network functions can be carried out in a self-organized way to combat the selfish behaviors. In dynamic spectrum allocation scenarios, the users' selfishness causes more challenges for efficient mechanism design, such as incentive-stimulation and price of anarchy [9], [11]. Therefore, novel spectrum allocation approaches need to be developed considering the dynamic nature of wireless networks and users' selfish behaviors.

Considering a general network scenario in which multiple primary users (legacy spectrum holders) and 
secondary users (unlicensed users) coexist, primary users attempt to sell unused spectrum resources to secondary users for monetary gains while secondary users try to acquire spectrum usage permissions from primary users to achieve certain communication goals, which generally introduces reward payoffs for them. In order to solve the above issues, we consider the spectrum sharing as multistage dynamic games and propose a dynamic pricing approach to optimize the overall spectrum efficiency, meanwhile, keeping the participating incentives of the users based on double-auction rules and coping with the budget constraints by dynamic programming. The main contributions of this paper are multi-fold. First, by modeling the spectrum sharing as a dynamic pricing game, we are able to quickly and accurately coordinate the spectrum allocation among primary and secondary users through a trading process to maximize the payoffs of both primary and secondary users. Further, we develop a belief system to assist greedy users update their strategies adaptive to the spectrum demand and supply changes, which not only approaches the theoretical optimal outcomes of the spectrum allocation problem but also substantially decreases the pricing overhead due to frequent bid/ask updates and message exchange. Third, by considering the budget constraints of the secondary users, the proposed dynamic pricing approach is able to further exploit the time diversity of spectrum resources.

The reminder of this paper is organized as follows: The system model of dynamic spectrum allocation is described in Section II. In Section III, we formulate the spectrum allocation as pricing games based on the system model. In Section IV, the belief-based dynamic pricing approach is proposed for the optimal spectrum allocation. The simulation studies are provided in Section V. Finally, Section VI concludes this paper.

\section{SYSTEM MOdEL}

We consider the wireless networks where multiple primary users and secondary users operate simultaneously in a wireless network, which may represent various network scenarios. For instance, the primary users can be the spectrum broker connected to the core network and the secondary users are the base stations equipped with cognitive radio technologies; or the primary users are the access points of a mesh network and the secondary users are the mobile devices. On one hand, every primary user has the license of using a certain spectrum range, which can be divided into non-overlapping orthogonal channels. Considering that the authorized spectrum of primary users may not be fully utilized over time, they prefer to lease the unused channels to the secondary users for monetary gains. On the other hand, since the unlicensed spectrums become more and more crowded, the secondary users may try to lease some unused channels from primary users for more communication gains by providing leasing payments.

In our system model, we assume all users are selfish and rational, that is, their objectives are to maximize their own payoffs, not to cause damage to other users. However, users are allowed to cheat whenever they believe cheating behaviors can help them to increase their payoffs. Generally speaking, in order to acquire the spectrum licenses from regulatory bodies such as FCC, the primary users have certain operating costs. With regard to secondary users, in order to have the rewards of achieving certain communication goals, they want to utilize more spectrum resources. The selfishness of both primary and secondary users will prevent them from revealing their private information such as acquisition costs or reward payoffs, which makes traditional spectrum allocation approaches not applicable under this scenario. Therefore, novel spectrum allocation approaches need to be developed which not only optimize the spectrum efficiency but also extract the private information from the selfish parties through certain mechanisms to assist the optimization of spectrum allocation.

Specifically, we consider the collection of the available spectrums from all primary users as a spectrum pool, which totally consists of $N$ non-overlapping channels. Assume there are $J$ primary users and $K$ secondary users, indicated by the set $\mathbf{P}=\left\{p_{1}, p_{2}, \ldots, p_{J}\right\}$ and $\mathbf{S}=\left\{s_{1}, s_{2}, \ldots, s_{K}\right\}$, respectively. We represent the channels authorized to primary user $p_{i}$ using a vector $\mathbf{A}_{i}=\left\{a_{i}^{j}\right\}_{j \in\left\{1,2, \ldots, n_{i}\right\}}$, where $a_{i}^{j}$ represents the channel index in the spectrum pool and $n_{i}$ is the total number of channels which belong to user $p_{i}$. Define $\mathbf{A}$ as the set of all the channels in the spectrum pool. Moreover, denote the acquisition costs of user $p_{i}$ 's channels as the vector $\mathbf{C}_{i}=\left\{c_{i}^{a_{i}^{j}}\right\}_{j \in\left\{1,2, \ldots, n_{i}\right\}}$, where the $j$ th element represents the acquisition cost of the $j$ th channel in $\mathbf{A}_{i}$. For simplicity, we write $c_{i}^{a_{i}^{j}}$ as $c_{i}^{j}$. As for secondary user $s_{i}$, we define her/his payoff vector as $\mathbf{V}_{i}=\left\{v_{i}^{j}\right\}_{j \in\{1,2, \ldots, N\}}$, where the $j$ th element is the reward payoff if this user successfully leases the $j$ th channel in the spectrum pool.

\section{Pricing Game Model}

In this paper, we model the dynamic spectrum allocation problem as a pricing game to study the interactions among the players, i.e., the primary and secondary users. Based on the discussion in the previous section, we are 
able to have the payoff functions of the players in our dynamic game. Specifically, if primary user $p_{i}$ reaches agreements of leasing all or part of her/his channels to secondary users, the payoff function of this primary user can be written as follows.

$$
U_{p_{i}}\left(\phi_{\mathbf{A}_{i}}, \alpha_{i}^{\mathbf{A}_{i}}\right)=\sum_{j=1}^{n_{i}}\left(\phi_{a_{i}^{j}}-c_{i}^{j}\right) \alpha_{i}^{a_{i}^{j}},
$$

where $\phi_{\mathbf{A}_{i}}=\left\{\phi_{a_{i}^{j}}\right\}_{j \in\left\{1,2, \ldots, n_{i}\right\}}$ and $\phi_{a_{i}^{j}}$ is the payment that user $p_{i}$ obtains from the secondary user by leasing the channel $a_{i}^{j}$ in the spectrum pool. Note that $\alpha_{i}^{\mathbf{A}_{i}}=$ $\left\{\alpha_{i}^{a_{i}^{j}}\right\}_{j \in\left\{1,2, \ldots, n_{i}\right\}}$ and $\alpha_{i}^{a_{i}^{j}} \in\{0,1\}$ which indicates if the $j$ th channel of user $p_{i}$ has been allocated to a secondary user or not. For simplicity, we denote $\alpha_{i}^{a_{i}^{j}}$ as $\alpha_{i}^{j}$. Similarly, the payoff function of secondary user $s_{i}$ can be modeled as follows.

$$
U_{s_{i}}\left(\phi_{\mathbf{A}}, \beta_{i}^{\mathbf{A}}\right)=\sum_{j=1}^{N}\left(v_{i}^{j}-\phi_{j}\right) \beta_{i}^{j},
$$

where $\phi_{\mathbf{A}}=\left\{\phi_{j}\right\}_{j \in\{1,2, \ldots, N\}}, \beta_{i}^{\mathbf{A}}=\left\{\beta_{i}^{j}\right\}_{j \in\{1,2, \ldots, N\}}$. Note that $\beta_{i}^{j} \in\{0,1\}$ illustrates if secondary user $s_{i}$ successfully leases the $j$ th channel in the spectrum pool or not. Hence, the strategies of the primary users and secondary users are actually defined by $\alpha_{i}^{\mathbf{A}_{i}}$ and $\beta_{i}^{\mathbf{A}}$, respectively.

Since the players may have conflict interests with each other, our dynamic spectrum sharing game can be modeled as a multi-stage non-cooperation game. To be specific, from the primary users' point of view, they want to earn the payments by leasing the unused channels which not only cover their spectrum acquisition costs but also gain as much extra payments as possible; from the secondary users' point of view, they aim to accomplish their communication goals by providing the least possible payments to lease the channels; while from the network designers' point of view, they attempt to maximize the network performance, which in our case is the spectrum efficiency. Therefore, the spectrum users involved in the spectrum sharing process construct a non-cooperative pricing game [11], [12]. Since the selfish users are their own authorities, they will not reveal their private information to others unless some mechanisms have been applied to guarantee that it is not harmful to disclose the private information. Generally, such non-cooperative game with incomplete information is complex and difficult to study as the players do not know the perfect strategy profile of others. But based on our game setting, the well-developed auction theory
[13] can be applied to formulate and analyze the pricing game.

In auction games [13], according to an explicit set of rules, the principles (auctioneers) determine resource allocation and prices on the basis of bids from the agents (bidders). In our spectrum allocation pricing game, the primary users can be viewed as the principles, who attempts to sell the unused channels to the secondary users. The secondary users are the bidders who compete with each other to buy the permission of using primary users' channels, by which they may gain extra payoffs for future use. In our pricing game, multiple sellers and buyers coexist, which indicates the double auction scenario. It means that not only the secondary users but also the primary users need to compete with each other to make the beneficial transactions possible by eliciting their willingness of the payments in the forms of bids or asks. Specifically, the double auction is one of the most common exchange mechanisms, used extensively in stock markets such as the New York Stock Exchange (NYSE) or commodity markets such as Chicago Merchandize Exchange (CME). The most important property of double auction mechanism is its high efficiency, which is still not fully understood in economic theory. Moreover, it can respond quickly to changing conditions of auction participants. However, in order to achieve the full efficiency of the double auction mechanism, a lot of messages need to be exchanged among the auction participants, which can be easily implemented by powerful central authorities in stock or commodity markets. It is worth noticing that in autonomous wireless networks either central authorities can be pre-assumed or the bandwidth of control channels is very limited. Therefore, we aims to develop an efficient pricing approach for spectrum allocation, which not only has the prevalence of the double auction mechanism but also uses simple message exchanges to quickly and accurately coordinate the spectrum sharing.

\section{Belief-Assisted DYNAMIC PRICING FOR EFFICIENT SPECTRUM ALLOCATION}

\section{A. Static Pricing Game and Competitive Equilibrium}

Assume that the available channels from the primary users are leased for usage of certain time period $T$. Also, we assume that the cost of the primary users and reward payoffs of the secondary users remain unchanged over this period. Before this spectrum sharing period, we define a trading period $\tau$, within which the users exchange their information of bids and asks to achieve agreements of spectrum usage. The time period $T+\tau$ is 
considered as one stage in our pricing game. We first study the interactions of the players in static pricing games. Note that the users' goals are to maximize their own payoff functions. As for the primary users, the optimization problem can be written as follows.

$$
\begin{array}{r}
O\left(p_{i}\right)=\max _{\phi_{\mathbf{A}_{i}}, \alpha_{i}^{\mathbf{A}_{i}}} U_{p_{i}}\left(\phi_{\mathbf{A}_{i}}, \alpha_{i}^{\mathbf{A}_{i}}\right), \quad \forall i \in\{1,2, \ldots, J\} \\
\text { s.t. } \quad U_{\hat{s}_{a_{i}^{j}}}\left(\left\{\phi_{-a_{i}^{j}}, \phi_{a_{i}^{j}}\right\}, \beta_{i}^{\mathbf{A}}\right) \geq U_{\hat{s}_{a_{i}^{j}}}\left(\left\{\phi_{-a_{i}^{j}}, \tilde{\phi}_{a_{i}^{j}}\right\}, \beta_{i}^{\mathbf{A}}\right), \\
\qquad \hat{s}_{a_{i}^{j}} \neq 0, a_{i}^{j} \in \mathbf{A}_{i} .
\end{array}
$$

where $\tilde{\phi}_{a_{i}^{j}}$ is any feasible payment and $\phi_{-a_{i}^{j}}$ is the payment vector excluding the element of the payment for the channel $a_{i}^{j}$. Note that $\hat{s}_{a_{i}^{j}}$ is defined as follows.

$$
\hat{s}_{a_{i}^{j}}= \begin{cases}s_{k} & \text { if } \beta_{k}^{a_{i}^{j}}=1, \\ 0 & \text { if } \beta_{k}^{a_{i}^{j}}=0, \forall k \in\{1,2, \ldots, K\} .\end{cases}
$$

Thus, (4) is the incentive compatible constraint [13]. It means that the secondary users have incentives to provide the optimal payment because they cannot have extra gains by cheating on the primary users. Similarly, the optimization problem can be written for the secondary users as follows.

$$
\begin{array}{r}
O\left(s_{i}\right)=\max _{\phi_{\mathbf{A}}, \beta_{i}^{\mathbf{A}}} U_{s_{i}}\left(\phi_{\mathbf{A}}, \beta_{i}^{\mathbf{A}}\right), \quad \forall i \in\{1,2, \ldots, K\} \\
\text { s.t. } \quad U_{\hat{p}_{j}}\left(\left\{\phi_{-j}, \phi_{j}\right\}, \beta_{i}^{\mathbf{A}}\right) \geq U_{\hat{p}_{j}}\left(\left\{\phi_{-j}, \tilde{\phi}_{j}\right\}, \beta_{i}^{\mathbf{A}}\right), \\
\hat{p}_{j} \neq 0, \beta_{i}^{j}=1 .
\end{array}
$$

where $\hat{p}_{j}$ is defined as

$$
\hat{p}_{j}= \begin{cases}p_{k} & \text { if } \beta_{i}^{j}=1, j \in \mathbf{A}_{k}, \alpha_{k}^{j}=1 \\ 0 & \text { otherwise, } \forall k \in\{1,2, \ldots, J\} .\end{cases}
$$

Similarly, (7) is the incentive compatible constraint for the primary users, which guarantees that the primary user will give the usage permission of their channels to the secondary users so that they can receive the optimal payments.

From (3) and (6), we can see that in order to obtain the optimal allocation and payments, a multi-objective optimization problem needs to be solved, which becomes extremely complicated due to our game setting that only involves incomplete information. Thus, in order to make this problem tangible, we analyze it from the game theory point of view. Generally speaking, game theory provides well-developed equilibrium concepts or optimality criteria to study the outcomes of games. For instance, Nash Equilibrium [12] is an important

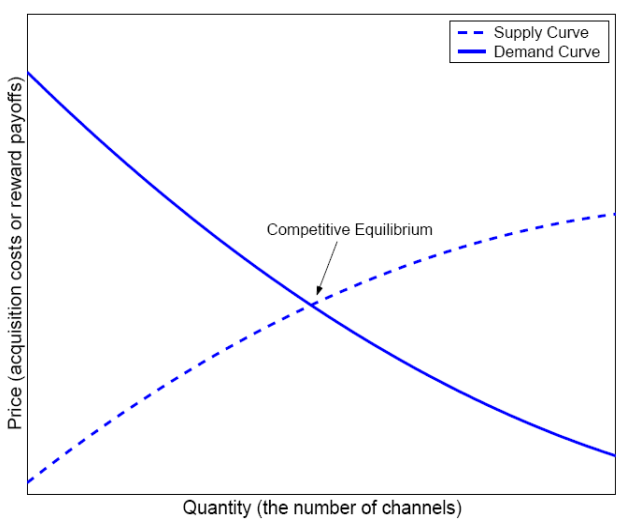

Fig. 1: Illustration of supply and demand functions.

concept to measure the outcome of a non-cooperation game, which is a set of strategies, one for each player, such that no selfish player has incentive to unilaterally change his/her action. In order to further measure the efficiency of game outcomes, Pareto Optimality [11] is defined such that a Pareto optimal outcome cannot be improved upon without hurting at least one player. Often, a Nash equilibrium is not Pareto optimal while Pareto optimal outcomes may not be sustained considering the selfishness of the players. Further, considering the double auction scenarios of our pricing game, Competitive Equilibrium (CE) [14] is a well-known theoretical prediction of the outcomes. It is the price at which the number of buyers willing to buy is equal to the number of sellers willing to sell. Alternatively, CE can also be interpreted as where the supply and demand match [13]. The supply function can be defined as the relationship between the acquisition costs of primary users and the number of corresponding channels; the demand function can be defined as the relationship between the reward payoffs of secondary users and the number of corresponding channels. We describe the supply and demand functions in Figure 1. Note that $\mathrm{CE}$ is also proved to be Pareto optimal in stationary double auction scenarios [15]. It is worth noting that in order to achieve the CE the traditional continuous bid/ask interactions among players will involve a great amount of message exchanges and require powerful centralized control, which may not be applicable to wireless networking scenarios due to the limited bandwidth of control channels.

\section{B. Belief-Assisted Dynamic Pricing}

Considering network dynamics due to mobility, channel variations or wireless traffic variations, the secondary users may have different reward payoffs of acquiring 
certain channels from primary users at different time stages. Specifically, since the secondary users can be mobile devices, they may move out the access range of certain channels and hence the corresponding reward payoffs $v_{i}^{j}$ are regarded as 0 . Or, the secondary users may face various channel fading conditions within different spectrum ranges or during different time periods, which changes their payoff values $v_{i}^{j}$ at different time stages. Moreover, the costs of primary users will also change over time due to network dynamics. For instance, if the legacy users themselves have larger spectrum demands, some legacy channels may not be available for leasing anymore, which actually indicates an infinite leasing cost of those channels in our pricing model. In brief, $c_{i}^{j}$ and $v_{i}^{j}$ need to be considered as random variables in dynamic scenarios, which we assume to satisfy the probability density functions (PDF) $f_{c}(c)$ and $f_{v}(v)$, respectively. Therefore, considering dynamic network conditions, we further model the spectrum sharing as a multi-stage dynamic pricing game. Let $\gamma$ be the discount factor of the multi-stage game. Based on (3) and (6), the objective functions for the primary users and secondary users can be rewritten as follows.

$$
\begin{gathered}
\tilde{O}\left(p_{i}\right)=\max _{\phi_{\mathbf{A}_{i}, t}, \alpha_{i, t}^{\mathbf{A}_{i}}} E_{c_{i}^{j}, v_{i}^{j}}\left[\sum_{t=1}^{\infty} \gamma^{t} \cdot U_{p_{i}, t}\left(\phi_{\mathbf{A}_{i}, t}, \alpha_{i, t}^{\mathbf{A}_{i}}\right)\right], \\
\tilde{O}\left(s_{i}\right)=\max _{\phi_{\mathbf{A}, t}, \beta_{i, t}^{\mathbf{A}}} E_{c_{i}^{j}, v_{i}^{j}}\left[\sum_{t=1}^{\infty} \gamma^{t} \cdot U_{s_{i}, t}\left(\phi_{\mathbf{A}, t}, \beta_{i, t}^{\mathbf{A}}\right)\right],
\end{gathered}
$$

where the subscript $t$ indicates the $t$ th stage of the multi-stage game. Generally speaking, there may exist some overall constraints of spectrum sharing such as each secondary user's total budget for leasing spectrum resources or each primary user's total available spectrum supply. Under these constraints, the above problem need to be further modeled as a dynamic programming process [16], [17] to obtain optimal sequential strategies by considering some state parameters such as the number of channels to be allocated at every stage or the residual monetary budget. However, the major difficulty of dynamic spectrum sharing lies in that how to efficiently and quickly update the spectrum sharing strategies adapt to the changing network conditions only based on local information. Therefore, in the following parts, we first focus on developing a belief-assisted dynamic pricing approach, which can not only approach CE outcomes but also responds quickly to networking dynamics while only introducing limited overhead. Then, the total budget constraint is taken into consideration and a dynamic programming approach is further proposed to obtain the optimal sequential strategies.

1) Belief-Assisted Dynamic Pricing: Since our pricing game belongs to the non-cooperation games with incomplete information [12], the players need to build up certain beliefs of other players' future possible strategies to assist their decision making. Considering that there are multiple players with private information in the pricing game and what directly affect the outcome of the game are the bid/ask prices, it is more efficient to define one common belief function based on the publicly observed bid/ask prices than generating specific belief of every other player's private information. Hence, enlightened by [14], we consider the primary/secondary users' beliefs as the ratio their bid/ask being accepted at different price levels. At each time during the dynamic spectrum sharing, the ratio of asks from primary users at $x$ that have been accepted can be written as follows.

$$
\tilde{r}_{p}(x)=\frac{\mu_{A}(x)}{\mu(x)},
$$

where $\mu(x)$ and $\mu_{A}(x)$ are the number of asks at $x$ and the number of accepted asks at $x$, respectively. Similarly, at each time during the dynamic spectrum sharing, the ratio of bids from secondary users at $y$ that have been accepted is

$$
\tilde{r}_{s}(y)=\frac{\eta_{A}(y)}{\eta(y)},
$$

where $\eta(y)$ and $\eta_{A}(y)$ are the number of bids at $y$ and the number of accepted bids at $y$, respectively. Usually, $\tilde{r}_{p}(x)$ and $\tilde{r}_{s}(y)$ can be accurately estimated if a great number of buyers and sellers are participating in the pricing at the same time. However, in our pricing game, only a relatively small number of players are involved in the spectrum sharing at the specific time. The beliefs, namely, $\tilde{r}_{p}(x)$ and $\tilde{r}_{s}(y)$ cannot be practically obtained so that we need to further consider using the historical bid/ask information to build up empirical belief values. Considering the characteristics of double auction, we have the following observations:

- If an ask $\tilde{x}<x$ is rejected, the ask at $x$ will also be rejected;

- If an ask $\tilde{x}>x$ is accepted, the ask at $x$ will also be accepted;

- If a bid $\tilde{y}>x$ is made, the ask at $x$ will also be accepted.

Based on the above observations, the players' beliefs can be further defined as follows using the past bid/ask information. 
Definition 1: Primary users' beliefs: for each potential ask at $x$, define

$\hat{r}_{p}(x)= \begin{cases}1 & x=0 \\ \frac{\sum_{w \geq x} \mu_{A}(w)+\sum_{w \geq x} \eta(w)}{\sum_{w \geq x} \mu_{A}(w)+\sum_{w \geq x} \eta(w)+\sum_{w \leq x} \mu_{R}(w)} & x \in(0, M) \\ 0 & x \geq M\end{cases}$

where $\mu_{R}(w)$ is the number of asks at $w$ that has been rejected, $M$ is a large enough value so that the asks greater than $M$ won't be accepted. Also, it is intuitive that the ask at 0 will be definitely accepted as no cost is introduced.

Definition 2: Secondary users' beliefs: for each potential bid at $y$, define

$\hat{r}_{s}(x)= \begin{cases}0 & y=0 \\ \frac{\sum_{w \leq y} \eta_{A}(w)+\sum_{w \leq y} \mu(w)}{\sum_{w \leq y} \eta_{A}(w)+\sum_{w \leq y} \mu(w)+\sum_{w \geq y} \eta_{R}(w)} & y \in(0, M) \\ 1 & y \geq M\end{cases}$

where $\eta_{R}(w)$ is the number of bids at $w$ that has been rejected. And, it is intuitive that the bid at 0 will not be accepted by any primary users.

Noting that it is too costly to build up beliefs on every possible bid or ask price, we can update the beliefs only at some fixed prices and use interpolation to obtain the belief function over the price space. Then, it is worth discussing the effect of the available public information on the efficiency of the above belief system. First, in the scenario that only local information is available to each user, the user updates the belief based on her/his own observed past bid/ask information, which results in more message exchanges to achieve the equilibrium price. Second, considering the broadcast nature of wireless channels, the neighbors' bid/ask information may be observed by the users, which can also be utilized to update the beliefs. In this scenario, the users may have part of the public information besides of their private information, which may accelerate their belief-updating pace and result in more efficient pricing process. Moreover, if the users have the access to all the public information such as ask/bid interactions through some centralized point, the above belief function is able to quickly reflect current supply and demand relationships.

Before using our defined belief functions to assist the strategy decisions, we first look at the Spread Reduction Rule (SRR) of double auction mechanisms. Generally, before the double auction pricing game converges to $\mathrm{CE}$, there may exist a gap between the highest bid and lowest ask, which is called the spread of double auction. The SRR states that any ask that is permissible must be lower than current lowest ask, i.e., outstanding ask [14], and then either each new ask results in an
TABLE I: Belief-assisted dynamic spectrum allocation

\begin{tabular}{l}
\hline 1. Initialize the users' beliefs and bids/asks \\
$\diamond$ The primary users initialize their asks as large values close to $M$ \\
and their beliefs as small positive values less than 1 ; \\
$\diamond$ The secondary users initialize their bids as small values close to 0 \\
and their beliefs as small positive values less than 1 . \\
\hline 2. Belief update based on local information: \\
Update primary and secondary users' beliefs \\
using (13) and (14), respectively \\
\hline 3. Optimal bid/ask update: \\
$\diamond$ Obtain the optimal ask for each primary user by solving (16); \\
$\diamond$ Obtain the optimal bid for each secondary user by solving (17). \\
\hline 4. Update leasing agreement and spectrum pool: \\
$\diamond$ If the outstanding bid is greater than or equal to the outstanding ask, \\
the leasing agreement will be signed between the corresponding users; \\
$\diamond$ Update the spectrum pool by removing the assigned channel. \\
\hline 5. Iteration: \\
If the spectrum pool is not empty, go back to Step 2 . \\
\hline
\end{tabular}

agreed transaction or it becomes the new outstanding ask. A similar argument can be applied to bids. By defining current outstanding ask and bid as $o x$ and $o y$, respectively, we let $\bar{r}_{p}(x)=\hat{r}_{p}(x) \cdot I_{[0, o x)}(x)$ for each $x$ and $\bar{r}_{s}(y)=\hat{r}_{s}(x) \cdot I_{(o y, M](y)}$ for each $y$, which are modified belief function considering the SRR. Note that $I_{(a, b)}(x)$ is defined as

$$
I_{(a, b)}(x)= \begin{cases}1 & \text { if } x \in(a, b) \\ 0 & \text { otherwise }\end{cases}
$$

By using the belief function $\bar{r}_{p}(x)$, the payoff maximization of selling the $i$ th primary user's $j$ th channel can be written as

$$
\max _{x \in(o y, o x)} E\left[U_{p_{i}}(x, j)\right],
$$

where $U_{p_{i}}(x, j)$ represents the payoff introduced by allocating the $j$ th channel when the ask is $x$, and then $E\left[U_{p_{i}}(x, j)\right]=\left(x-c_{i}^{j}\right) \cdot \bar{r}_{p}(x)$. Similarly, as for the secondary user $s_{i}$, the payoff maximization of leasing the $j$ th channel in the spectrum pool can be written as

$$
\max _{y \in(o y, o x)} E\left[U_{s_{i}}(y, j)\right]
$$

where $U_{s_{i}}(y, j)$ represents the payoff introduced by leasing the $j$ th channel in the spectrum pool when the bid is $y$, and then $E\left[U_{s_{i}}(y, j)\right]=\left(v_{i}^{j}-y\right) \cdot \bar{r}_{s}(y)$. Therefore, by solving the optimization problem for each primary and secondary user using (16) and (17), respectively, primary and secondary users can make the optimal decision of spectrum allocation at every stage conditional on dynamic spectrum demand and supply. Based on the above discussions, we illustrate our belief-assisted dynamic pricing algorithm for spectrum allocation in Table I. 
2) Dynamic Pricing with Budget Constraints: Based on the belief-assisted dynamic pricing algorithm developed above, in this part we further consider the optimal spectrum allocation when each secondary user is constrained by a total monetary budget for leasing spectrum usage. Note that the spectrum allocation problem can be similarly solved when the overall constraints exist for primary users.

Considering the budget constraints of secondary users, we rewrite their optimization objectives as follows.

$$
\begin{aligned}
\hat{O}\left(s_{i}\right)= & \max _{\phi_{A, t}, \beta_{i, t}^{A}, \psi_{i}} E_{c_{i}^{j}, v_{i}^{j}}\left[\sum_{t=1}^{\infty} \gamma^{t} \cdot U_{s_{i}, t}\left(\phi_{A, t}, \beta_{i, t}^{A}, \tilde{\psi}_{i, t}\right)\right], \\
\text { s.t. } \quad & U_{\hat{p}_{j}, t}\left(\left\{\phi_{-j, t}, \phi_{j, t}\right\}\right) \geq U_{\hat{p}_{j}, t}\left(\left\{\phi_{-j, t}, \tilde{\phi}_{j, t}\right\}\right),(19) \\
& \sum_{t=1}^{\infty} \psi_{t} \leq B_{i} .
\end{aligned}
$$

where $\psi_{i}=\left\{\psi_{i, t}\right\}_{t \in\{1,2, \ldots, \infty\}}$ and $\psi_{i, t}$ is the total monetary payment used during the $t$ th stage for the $i$ th secondary user leasing the channels. Moreover, $B_{i}$ is the $i$ th secondary user' total budget. Note that $\tilde{\psi}_{i, t}=$ $B_{i}-\sum_{\tau=1}^{\tau=t-1} \psi_{i, \tau}$, which is the residual budget at the $t$ th stage and can be considered as a state parameter. Hence, (19) and (20) are the incentive compatible constraint and total budget constraint, respectively. As it is difficult to directly solve (18), we study the dynamic programming approach to simplify the multistage optimization problem.

Define the value function $Q_{s_{i}, t}\left(\tilde{\psi}_{i}\right)$ as the $i$ th secondary user's maximum expected payoff obtainable from periods $t, t+1, \ldots, \infty$ given that the monetary budget left is $\tilde{\psi}_{i}$. Simplifying (18) using the Bellman equation [16], we have the maximal expected payoff $Q_{s_{i}, t}\left(\tilde{\psi}_{i}\right)$ written as follows.

$$
\begin{array}{r}
Q_{s_{i}, t}\left(\tilde{\psi}_{i}\right)=\max _{\phi_{\mathbf{A}, t}, \beta_{i, t}^{\mathbf{A}}, \psi_{i}}\left\{E _ { c _ { i } ^ { j } , v _ { i } ^ { j } } \left[U_{s_{i}, t}\left(\phi_{\mathbf{A}, t}, \beta_{i, t}^{\mathbf{A}}, \tilde{\psi}_{i}\right)+\right.\right. \\
\left.\left.\gamma \cdot Q_{s_{i}, t+1}\left(\tilde{\psi}_{i}-\psi_{i, t}\right)\right]\right\}, \\
\text { s.t. } U_{\hat{p}_{j}, t}\left(\left\{\phi_{-j, t}, \phi_{j, t}\right\}\right) \geq U_{\hat{p}_{j}, t}\left(\left\{\phi_{-j, t}, \tilde{\phi}_{j, t}\right\}\right) .
\end{array}
$$

The boundary conditions for the above dynamic programming problem are

$$
Q_{s_{i}, \infty}\left(\tilde{\psi}_{i}\right)=0, \quad \tilde{\psi}_{i} \in\left(0, B_{i}\right] .
$$

Note that the first term on the right hand side (RHS) of (21) represents the payoff at current stage and the second term on the RHS of (21) represents the future payoff obtained after the $t$ th stage give the budget state $\tilde{\psi}_{i}-\psi_{i, t}$. Further, applying the principle of optimality in
[16], the spectrum sharing configuration $\left\{\phi_{\mathbf{A}, t}, \beta_{i, t}^{\mathbf{A}}, \psi_{i}\right\}$ that achieves the maximum in (21) given $\tilde{\psi}_{i}, t$ and the statistics of $c_{i}^{j}, v_{i}^{j}$ is also the optimal solution for the overall optimization problem (18).

In order to obtain $Q_{s_{i}, t}\left(\tilde{\psi}_{i}\right)$, the maximal payoff of one stage needs to be first derived for different residual budget values $\tilde{\psi}_{i}$. The difference of the current payoff function in (18) and the one-stage payoff function in (6) lies in that the applied budget constraint affects the outcomes of the pricing game. For instance, even though both the primary users and secondary users can achieve higher payoffs by assigning a channel to user $s_{i}$, the user $s_{i}$ may not have enough budgets to lease this channel. Thus, the algorithm in Table I cannot be directly applied here for optimal spectrum sharing. We need to modify the bid update step as follows: user $s_{i}$ updates his/her bid by $\min \left\{\tilde{\psi}_{i}, y\right\}$, where $y$ is obtained from (17). Note that it is highly complicated to derive the close-form solution for the one-stage payoff function in (18) [13], [15].Thus, we use simulation to approximate it for different residual budget values, which proceeds as follows: Generate a large number of samples of the secondary and primary users with reward payoffs and costs satisfying $f_{v}(v)$ and $f_{c}(c)$, respectively. Using the above modified version of the algorithm in Table I, calculate the average one-stage payoffs given different $\tilde{\psi}$ based on the outcomes of the spectrum allocation samples.

By using the numerical results of the one-stage payoff function, we then derive $Q_{s_{i}, t}\left(\tilde{\psi}_{i}\right)$ using dynamic programming methods. Considering infinite spectrum allocation stages, the maximum payoff $Q_{s_{i}, t}\left(\tilde{\psi}_{i}\right)$ in (21) can be written as follows.

$$
\begin{array}{r}
Q_{s_{i}}^{*}\left(\tilde{\psi}_{i}\right)=\max _{\phi_{\mathbf{A}, t}, \beta_{i, t}^{\mathbf{A}}, \psi_{i}}\left\{E _ { c _ { i } ^ { j } , v _ { i } ^ { j } } \left[U_{s_{i}, t}\left(\phi_{\mathbf{A}, t}, \beta_{i, t}^{\mathbf{A}}, \tilde{\psi}_{i}\right)+\right.\right. \\
\left.\left.\gamma \cdot Q_{s_{i}}^{*}\left(\tilde{\psi}_{i}-\psi_{i, t}\right)\right]\right\},
\end{array}
$$

or, equivalently, $Q_{s_{i}}^{*}=\mathcal{T} Q_{s_{i}}^{*}$, where $\mathcal{T}$ is the operator updating $Q_{s_{i}}^{*}$ using (24). Let $\mathcal{S}$ be the feasible set of the state parameter. The convergence proposition of the dynamic programming algorithm [16] can be applied here, which states that: for any bounded function $Q$ : $\mathcal{S} \rightarrow \mathcal{R}$, the optimal payoff function satisfies $Q^{*}(x)=$ $\lim _{p \rightarrow \infty}\left(\mathcal{T}^{p} Q\right)(x), \forall x \in \mathcal{S}$. As $Q_{s_{i}}\left(\tilde{\psi}_{i}\right)$ is bounded in our algorithm, we are able to apply the value iteration method to approximate the optimal $Q_{s_{i}}\left(\tilde{\psi}_{i}\right)$, which proceeds as follows: Start from some initial function for $Q_{s_{i}}\left(\tilde{\psi}_{i}\right)$ as $Q_{s_{i}}^{0}\left(\tilde{\psi}_{i}\right)=g(x)$, where the superscript stands for the iteration number. Then, iteratively update $Q_{s_{i}}\left(\tilde{\psi}_{i}\right)$ by letting $Q_{s_{i}}^{p+1}\left(\tilde{\psi}_{i}\right)=\left(\mathcal{T} Q_{s_{i}}^{p}\right)\left(\tilde{\psi}_{i}\right)$. The iteration process 


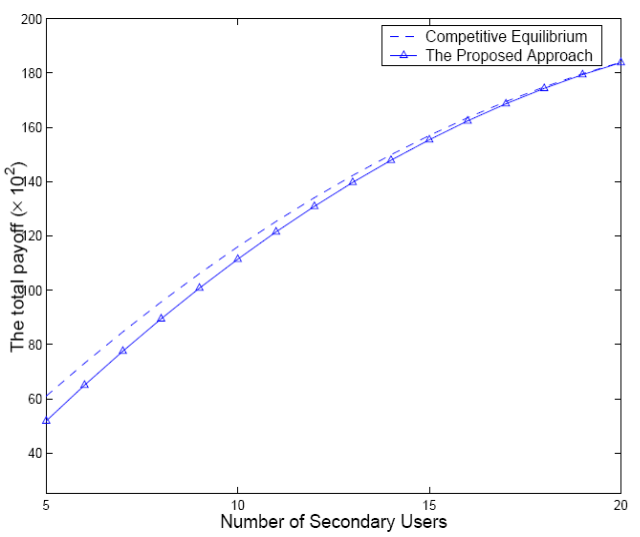

Fig. 2: Comparison of the total payoff for the proposed scheme and theoretical Competitive Equilibrium.

ends until $\left|Q_{s_{i}}^{p+1}\left(\tilde{\psi}_{i}\right)-Q_{s_{i}}^{p}\left(\tilde{\psi}_{i}\right)\right| \leq_{\tilde{\tau}} \epsilon$, for all $\tilde{\psi}_{i} \in \mathcal{S}$, where $\epsilon$ is the error bound for $Q_{s_{i}}^{*}\left(\tilde{\psi}_{i}\right)$.

Intuitively, the basic idea behind our dynamic pricing approach for spectrum allocation with budget constraints can be explained as follows: Considering the overall budget constraints, the users make their spectrum sharing decisions not only based on their current payoffs but also based on expected future payoffs. Specifically, if the competition for spectrum resources is high at current stage, the users prefer to save their monetary budgets for future usage, which will yield higher overall payoffs for the users. Therefore, by using our proposed dynamic pricing approach, the spectrum allocation can be optimized not only in the space and frequency domains but also in the the time domain.

\section{Simulation Results}

In this section, we evaluate the performance of the proposed belief-assisted dynamic spectrum sharing approach in wireless networks. Considering a wireless network covering $100 \times 100$ area, we simulate $J$ primary users by randomly placing them in the network. These primary users can be the base stations serving for different wireless network operators or different access points in a mesh network. Here we assume the primary users' locations are fixed and their unused channels are available to the secondary users within the distance of 50. Then, we randomly deploy $K$ secondary users in the network, which are assumed to be mobile devices. The mobility of the secondary users is modeled using a simplified random waypoint model [18], where we assume the "thinking time" at each waypoint is close to the effective duration of one channel-leasing agreement, the waypoints are uniformly distributed within the

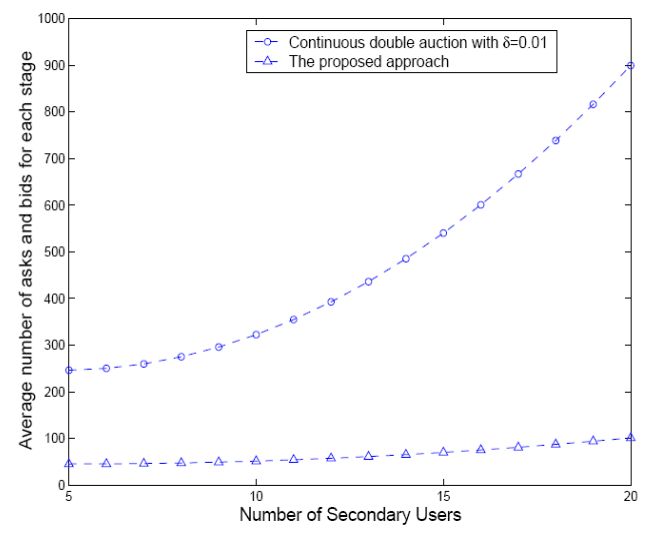

Fig. 3: Comparison of the overhead between the proposed scheme and continuous double auction scheme.

distance of 10 , and the traveling time is much smaller than the "thinking time". Let the cost of an available channel in the spectrum pool be uniformly distributed in $[10,30]$, the reward payoff of leasing one channel be uniformly distributed in $[20,40]$. If a channel is not available to some secondary users, let the corresponding reward payoffs of this channel be 0 . Note that $J=5$ and $10^{3}$ pricing stages have been simulated. Let $n_{i}=$ $4, \forall i \in\{1,2, \ldots, J\}$ and $\gamma=0.99$.

We first focus our simulation studies on dynamic spectrum sharing without budget constraints, which can be used to illustrate the efficiency of the proposed belief-assisted pricing algorithm for spectrum allocation. In our simulation, the local bid/ask information within the transmission range of each node is used for belief construction and update. In Figure 2, we compare the total payoff of all users of our proposed approach with that of the theoretical CE outcomes for different number of secondary users. It can be seen from this figure that the performance loss of our approach is very limited compared to that of the theoretical optimal solutions. Moreover, when the number of secondary users increases, our approach is able to approach the optimal CE. It is because that the belief function reflects the spectrum demand and supply more accurately when more users are involved in spectrum sharing.

Now we study the overhead of our pricing approach. Here we measure the pricing overhead by showing the average number of bids and asks for each stage. In Figure 3, the overhead of our pricing approach is compared to that of the traditional continuous double auction when the same total payoff is achieved. Assume the minimal bid/ask step $\delta$ of the continuous double auction to be 0.01 . It can be seen from the figure 


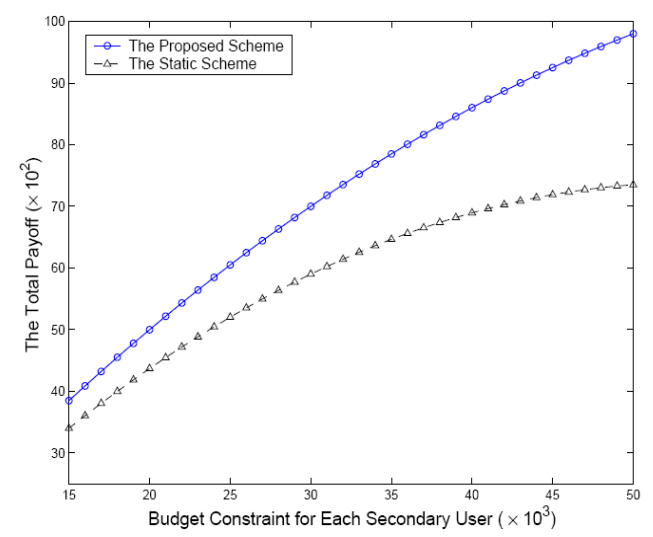

Fig. 4: Comparison of the total payoffs of the proposed scheme with those of the static scheme.

that our approach substantially decreases the pricing communication overhead. Note that when decreasing the overhead, our proposed approach may introduce extra complexity to update the beliefs.

Then, we study the dynamic spectrum allocation when each secondary user is constrained by his/her monetary budget. For comparison, we define a static scheme in which the secondary users make their spectrum-leasing decisions without considering their budget limits. Without loss of generality, we assume that the budget constraints for the secondary users are the same. In Figure 4, we compare the total payoffs of our proposed dynamic programming scheme with those of the static scheme for different budget constraints. It can be seen from the figure that our proposed scheme achieves significant performance gains over the static scheme when the budget constraints are taken into consideration. Also, when the budget limits increase, the proposed scheme achieves higher gain by further exploiting the time diversity.

\section{CONClusions AND Future Work}

In this paper, we have studied dynamic pricing for efficient spectrum allocation in wireless networks with selfish users. We model the dynamic spectrum allocation as a multi-stage game and propose a belief-assisted dynamic pricing approach to maximize the users' payoffs while providing them the participating incentives via double auction rules. Further, the dynamic pricing under the budget constraints of secondary users is analyzed using dynamic programming. Simulation results show that the proposed scheme can approach the optimal performances by only using limited overhead. Moreover, the time diversity of spectrum resources can be fully exploited when budget constraints exist.
There are several avenues for future research. We intend to further perform the equilibrium analysis of the solution obtained by the proposed dynamic spectrum allocation scheme. Also, we would like to study how to adapt our scheme to mesh, cellular or combined networks under practical constraints caused by the nature of wireless networking. Further, security issues need to be extensively investigated for dynamic spectrum allocation. Not only the security threats caused by the designed mechanism itself such as bidding ring problems [13] need to be considered, but also the threats due to the limitations of wireless systems and radio interface.

\section{REFERENCES}

[1] FCC, "Spectrum policy task force report," FCC Document ET Docket No. 02-135, November 2002.

[2] FCC, "Facilitating opportunities for flexible, efficient, and reliable spectrum use employing cognitive radio technologies: notice of proposed rule making and order," FCC Document ET Docket No. 03-108, December 2003.

[3] R. J. Berger, "Open spectrum: a path to ubiquitous connectivity," FCC ACM Queue 1, 3, May 2003.

[4] J. M. Peha, "Approaches to spectrum sharing," IEEE Communications Magazine, vol. 43, pp. 10-12, Feburary 2005.

[5] M. M. Buddhikot, "Dimsumnet: new directions in wireless networking using coordinated dynamic spectrum access," in Proc. of IEEE WoWMoM'05, 2005.

[6] C. Peng, H. Zheng, and B. Y. Zhao, "Utilization and fairness in spectrum assignment for opportunistic spectrum access," to appear in Mobile Networks and Applications (MONET), 2006.

[7] L. Cao and H. Zheng, "Distributied spectrum allocation via local bargaining," in Proc. of IEEE DySpan, 2005.

[8] R. Etkin, A. Parekh, and D. Tse, "Spectrum sharing for unlicensed bands," in Proc. of IEEE DySpan, 2005.

[9] M. H. Halldorson, J. H. Halpern, L. Li, V. S. Mirrokni, "On spectrum sharing games," in Proc. of ACM Symposium on Principle of Distributed Computing (PODC), 2004.

[10] M. Felegyhazi and J. P. Hubaux, "Wireless operators in a shared spectrum," in Proc. of IEEE INFOCOM'06, 2006.

[11] D. Fudenberg and J. Tirole, Game Theory, The MIT Press, Cambridge, Massachusetts, 1991.

[12] M. J. Osborne and A. Rubinstein, A Course in Game Theory, The MIT Press, Cambridge, Massachusetts, 1994.

[13] V. Krishna, Auction Theory, Academic Press, 2002.

[14] S. Gjerstad and J. Dickhaut, "Price formation in double auctions," Games and Economic Behavior, vol. 22, pp. 1-29, 1998.

[15] L. Hurwicz, R. Radner, and S. Reiter, "A stochastic decentralized resource allocation process: Part i," Econometrica, vol. 43, pp. 363-393, 1975.

[16] D. Bertsekas, Dynamic Programming and Optimal Control, vol. 1,2, Athena Scientific, Belmont, MA, Second edition, 2001.

[17] Z. Ji, W. Yu, and K. J. R. Liu, "An optimal dynamic pricing framework for autonomous mobile ad hoc networks," in Proc. of IEEE INFOCOM'O6, 2006.

[18] D. B. Johnson and D. A. Maltz, "Dynamic source routing in ad hoc wireless networks, mobile computing," IEEE Transactions on Mobile Computing, pp. 153-181, 2000. 